\title{
TUBERCULOSIS EN EL PERÚ: SITUACIÓN EPIDEMIOLÓGICA, AVANCES Y DESAFÍOS PARA SU CONTROL
}

\author{
Valentina Alarcón ${ }^{1, a}$, Edith Alarcón²,b ${ }^{2}$ Cecilia Figueroa ${ }^{1, c}$, Alberto Mendoza-Ticona $^{1, d}$
}

\begin{abstract}
RESUMEN
La tuberculosis (TB) es la primera causa de muerte por un agente infeccioso en el mundo, la incidencia en la población viene disminuyendo muy lentamente y la resistencia a los medicamentos es actualmente considerada como una crisis internacional. En el Perú, la reciente Ley de Prevención y Control de la TB en el Perú (Ley 30287), declara de interés nacional la lucha contra la TB. En los últimos años, la Estrategia Sanitaria Nacional de Prevención y Control de la Tuberculosis (ESNPCT) del Ministerio de Salud (MINSA), ha obtenido avances significativos en el control de esta enfermedad; sin embargo, aún persisten desafíos que deben ser abordados. El presente artículo revisa la situación epidemiológica de la TB en el Perú, sistematiza los avances logrados durante la gestión del equipo de la ESNPCT entre los años 2011 y 2015 desde el abordaje biomédico, de gestión pública y en las determinantes sociales de la salud, además, plantea desafíos para lograr el control de la TB, en el marco de la Ley 30287 y la estrategia "Fin de la TB" de la Organización Mundial de la Salud (OMS).
\end{abstract}

Palabras clave: Tuberculosis; Epidemiología; Administración de los Senvicios de Salud; Salud Pública; Perú (Fuente: DeCS BIREME).

\section{TUBERCULOSIS IN PERU: EPIDEMIOLOGICAL SITUATION, PROGRESS AND CHALLENGES FOR ITS CONTROL}

\begin{abstract}
Tuberculosis (TB) is the first cause of death by an infectious agent in the world, the incidence in the population is declining very slowly and drug resistance is currently considered an international crisis. In Peru, the recent TB Prevention and Control Act in Peru (Law 30287) declares the fight against TB of national interest. In recent years, the Ministry of Health's (MINSA) National Health Strategy for the Prevention and Control of Tuberculosis (ESNPCT) has achieved significant progress in the control of this disease; however, challenges still remain to be addressed. This article reviews the epidemiological situation of TB in Peru, systematizes the progress achieved during the management of the ESNPCT team between the years 2011 and 2015 from the biomedical approach, public management and social determinants of health, also posing challenges to achieving TB control under law 30287 and the "End of TB" strategy of the World Health Organization (WHO).
\end{abstract}

Key words: Tuberculosis; Epidemiology; Health Services Administration; Public health; Peru (Source: MeSH NLM).

\section{INTRODUCCIÓN}

La tuberculosis (TB) es una enfermedad infectocontagiosa, prevenible, curable y con un importante componente social ${ }^{(1)}$. Según estimaciones de la Organización Mundial de la Salud (OMS), la incidencia anual de la TB a nivel mundial está disminuyendo lentamente, alrededor de $1,5 \%$ desde el año 2000; sin embargo, el número absoluto de casos de TB se viene incrementando. Para el año 2015, se estima que se produjeron: 10,4 millones de casos de TB, 580 mil casos de TB multidrogorresistente (TB MDR), 1,2 millones de nuevos casos de TB/VIH y 1,8 millones de defunciones, por lo que el Mycobacterium tuberculosis se ha convertido en el agente infeccioso que más muertes ocasiona, por encima del VIH y la malaria (2).

Como respuesta global a la epidemia de la TB, la OMS ha elaborado la estrategia "Fin de la Tuberculosis" cuyas metas al 2035, con respecto al año 2015, son: i) reducir en un $95 \%$ de muertes por TB; ii) reducir la tasa de incidencia de TB en un 90\% (lograr una tasa < 10 casos por cada 100 mil habitantes), y iii) lograr cero familias afectadas por costos catastróficos por TB (se

\footnotetext{
Estrategia Sanitaria Nacional Prevención y Control de la Tuberculosis, Ministerio de Salud. Lima, Perú

Unión Internacional Contra la Tuberculosis y Enfermedades Respiratorias. Lima, Perú.

Médico cirujano; ${ }^{\mathrm{b}}$ licenciada en Enfermería, maestra en Salud Pública; ${ }^{\mathrm{c}}$ ingeniera de Sistemas; ${ }^{\mathrm{d}}$ médico infectólogo, maestro en Epidemiología Clínica Recibido: 12/09/2016 Aprobado: 22/03/2017 En línea: 28/06/2017
} 
refiere al pago directo por el usuario de gastos médicos y no médicos y costos indirectos que excedan un umbral dado de la renta del hogar, por ejemplo, el 20\%) ${ }^{(3)}$. Los tres pilares para el logro de las metas de la estrategia "Fin de la Tuberculosis", son: Pilar 1: Prevención y atención integral de la TB centrada en el paciente; Pilar 2: Políticas audaces y sistemas de soporte, y Pilar 3: Investigación e innovación intensificada ${ }^{(4,5)}$.

En el Perú, la Ley 30287, Ley de Control y Prevención de la Tuberculosis en el Perú ${ }^{(6)}$ y su Reglamento (Decreto Supremo 021-2016), (7) declara de interés nacional la lucha contra la TB en el país, logrando, de esta forma, que el control de la TB sea una política de Estado, independiente a los gobiernos de turno. Las personas afectadas por TB en el Perú son diagnosticadas y tratadas de manera gratuita por las instituciones del sistema de salud del país: el $73 \%$ se atienden en el MINSA, el $19 \%$ en la Seguridad Social (ESSALUD), el $7 \%$ en el Instituto Nacional Penitenciario (INPE) y el $1 \%$ en las Sanidades de la Policía Nacional y las Fuerzas Armadas. La atención privada de la TB es limitada, dada la prohibición de la venta libre de medicamentos anti-TB en el Perú. Los pacientes diagnosticados en el sector privado son atendidos en coordinación con EsSalud y el MINSA, tanto las formas sensibles como las resistentes de TB.

En el presente artículo se desarrolla la situación epidemiológica de la TB en el Perú al 2015, los avances en su control, de acuerdo a tres enfoques: biomédico, de gestión pública y de determinantes sociales de la salud, y plantea desafíos para hacer frente a la TB en el marco de la Ley 30287 y la estrategia "Fin de la Tuberculosis".

\section{SITUACIÓN EPIDEMIOLÓGICA DE LA TB EN EL PERÚ}

La TB en el Perú ocupa el décimo quinto lugar de las causas de muerte ${ }^{(8)}$, y el vigésimo séptimo puesto de carga de enfermedad medida por años de vida saludable perdidos (AVISA) ${ }^{\left.{ }^{9}\right)}$. Afecta, predominantemente, a los estratos sociales más pobres de las grandes ciudades del país ${ }^{(10)}$. Las tasas notificadas de incidencia (casos nunca tratados por cada 100 mil habitantes) y de morbilidad total (nuevos y antes tratados por cada 100 mil habitantes) han disminuido entre 2 a 3\% por año entre los años 2011 a 2015 , de 97,4 a 87,6 en incidencia y de 109,7 a 99,5 en morbilidad. En la Figura 1, se muestra la tendencia de los principales indicadores epidemiológicos que son evaluados cada año por la Estrategia Sanitaria Nacional Prevención y Control de laTuberculosis (ESNPCT) entre los años 2008 y 2015: tasa de incidencia, tasa de morbilidad, tasa de incidencia de TB pulmonar frotis positivo y tasa de defunciones durante el tratamiento.

En el año 2015 se notificaron 30988 casos de TB, y la tasa de incidencia ha sido la menor reportada en los últimos 25 años, con 87,6 casos nuevos de TB por cada 100 mil habitantes. La ESNPCT notifica las defunciones durante el tratamiento (por cualquier causa), y se calcula como un sustituto de la tasa de mortalidad por cada 100 mil habitantes, la cual se mantiene alrededor de 3,7 en los últimos 3 años. Es importante destacar que la identificación

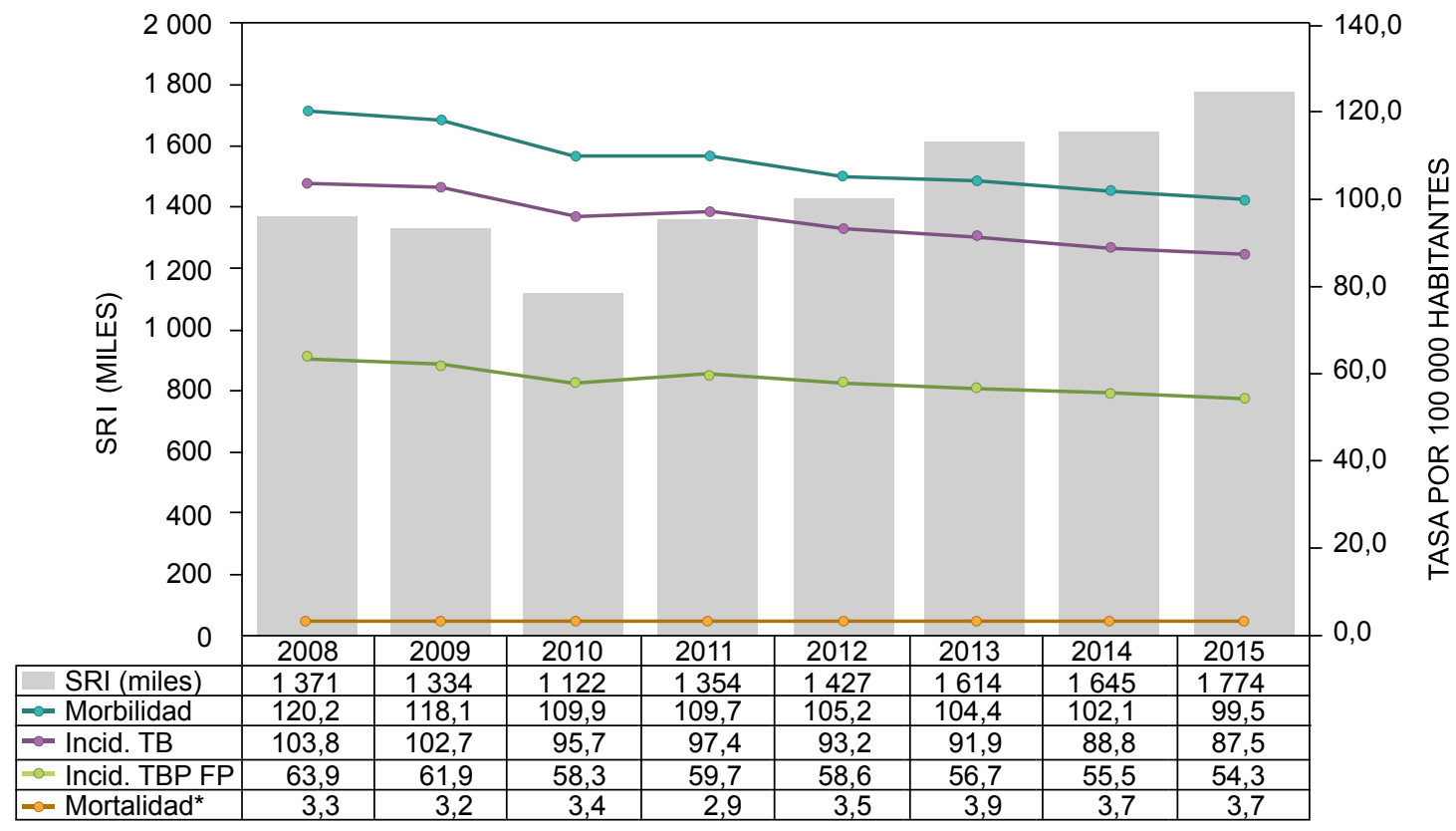

*Expresada en número de defunciones durante el tratamiento por 100 mil habitantes

Figura 1. Principales indicadores epidemiológicos y número de sintomáticos respiratorios identificados (SRI), Perú 2008- 2015. Fuente: ESNPCT - MINSA 
de sintomáticos respiratorios (SR), en números absolutos, se ha incrementado sostenidamente en los últimos 5 años, alcanzando su máximo valor en el 2015 con 1774000 SR identificados.

Sin embargo, la OMS estima que en el Perú se produce un mayor número de casos de TB de los que son notificados. Para el año 2015, la OMS estimó que se produjeron 37 mil casos de TB, con una tasa de incidencia de 119 casos por 100 mil habitantes y 2500 defunciones por TB. La diferencia entre las estimaciones y lo notificado es constante y frecuente para los países en desarrollo. La OMS basa sus estimaciones en proyecciones de estudios poblacionales como medición de prevalencia de TB, por lo que una mejor estimación podría lograrse haciendo estudios de prevalencia de TB en Perú con inferencia nacional, pero estos estudios son costosos y complejos.
En la Tabla 1 se presentan la población, el número total de casos y los indicadores epidemiológicos de la TB según regiones de salud para el año 2015. El $80 \%$ de los casos en el año 2015 fueron reportados por diez regiones de salud (en orden decreciente): Lima Metropolitana (Lima Este, Lima Ciudad y Lima Sur), Callao, La Libertad, Loreto, Ica, Lima Provincias, Junín, Lambayeque, Arequipa y Ucayali. Lima Metropolitana y la Región Callao notificaron el $59,3 \%$ de todos los casos de TB a nivel nacional en el año 2015.

La distribución de casos nuevos de TB en el año 2015, de acuerdo al género y a los grupos de edad, se muestra en la Tabla 2. De los 27299 casos nuevos notificados en el año 2015, 16680 (61,1\%) fueron varones. En el grupo de menores de 15 años no hay diferencia en cuanto al género. El grupo de edad entre 15 y 24 años contribuye con la mayor

Tabla 1. Casos y tasas poblacionales de tuberculosis, según regiones de salud, Perú año 2015

\begin{tabular}{|c|c|c|c|c|c|c|c|c|c|}
\hline \multirow[b]{2}{*}{ Región } & \multirow[b]{2}{*}{ Población } & \multicolumn{2}{|c|}{ Morbilidad } & \multicolumn{2}{|c|}{ Incidencia } & \multicolumn{2}{|c|}{ Incid. TBP FP } & \multicolumn{2}{|c|}{ Mortalidad } \\
\hline & & Casos & Tasa & Casos & Tasa & Casos & Tasa & Casos & Tasa \\
\hline Callao & 1010315 & 2499 & 247,3 & 2204 & 218,1 & 1271 & 125,8 & 129 & 12,8 \\
\hline Lima Este & 2601751 & 5521 & 212,2 & 4915 & 188,9 & 2929 & 112,6 & 126 & 4,8 \\
\hline Lima Ciudad & 4037774 & 7909 & 195,9 & 6657 & 164,9 & 4300 & 106,5 & 218 & 5,4 \\
\hline Madre de Dios & 137316 & 222 & 161,7 & 208 & 151,5 & 160 & 116,5 & 11 & 8,0 \\
\hline Ucayali & 495522 & 734 & 148,1 & 649 & 131,0 & 459 & 92,6 & 33 & 6,7 \\
\hline Loreto & 1039372 & 1392 & 133,9 & 1266 & 121,8 & 840 & 80,8 & 61 & 5,9 \\
\hline Tacna & 341838 & 432 & 126,4 & 385 & 112,6 & 247 & 72,3 & 6 & 1,8 \\
\hline Ica & 787170 & 901 & 114,5 & 761 & 96,7 & 463 & 58,8 & 35 & 4,4 \\
\hline Lima Sur & 2250594 & 2449 & 108,8 & 2128 & 94,6 & 1361 & 60,5 & 71 & 3,2 \\
\hline Moquegua & 180477 & 163 & 90,3 & 153 & 84,8 & 90 & 49,9 & 7 & 3,9 \\
\hline Lima Provincia & 948132 & 866 & 91,3 & 736 & 77,6 & 426 & 44,9 & 48 & 5,1 \\
\hline La Libertad & 1859640 & 1425 & 76,6 & 1270 & 68,3 & 804 & 43,2 & 56 & 3,0 \\
\hline Lambayeque & 1260650 & 802 & 63,6 & 718 & 57,0 & 464 & 36,8 & 30 & 2,4 \\
\hline Junín & 1350783 & 833 & 61,7 & 760 & 56,3 & 429 & 31,8 & 53 & 3,9 \\
\hline Arequipa & 1287205 & 750 & 58,3 & 672 & 52,2 & 376 & 29,2 & 38 & 3,0 \\
\hline Ancash & 1148634 & 646 & 56,2 & 577 & 50,2 & 368 & 32,0 & 36 & 3,1 \\
\hline Huánuco & 860537 & 452 & 52,5 & 432 & 50,2 & 313 & 36,4 & 12 & 1,4 \\
\hline San Martín & 840790 & 421 & 50,1 & 389 & 46,3 & 220 & 26,2 & 36 & 4,3 \\
\hline Cusco & 1316729 & 608 & 46,2 & 586 & 44,5 & 348 & 26,4 & 45 & 3,4 \\
\hline Tumbes & 237685 & 99 & 41,7 & 87 & 36,6 & 62 & 26,1 & 9 & 3,8 \\
\hline Ayacucho & 688657 & 244 & 35,4 & 227 & 33,0 & 144 & 20,9 & 17 & 2,5 \\
\hline Pasco & 304158 & 101 & 33,2 & 97 & 31,9 & 62 & 20,4 & 2 & 0,7 \\
\hline Piura & 1844129 & 586 & 31,8 & 546 & 29,6 & 297 & 16,1 & 18 & 1,0 \\
\hline Amazonas & 422629 & 123 & 29,1 & 119 & 28,2 & 64 & 15,1 & 0 & 0,0 \\
\hline Puno & 1415608 & 420 & 29,7 & 396 & 28,0 & 225 & 15,9 & 42 & 3,0 \\
\hline Apurímac & 458830 & 92 & 20,1 & 84 & 18,3 & 42 & 9,2 & 8 & 1,7 \\
\hline Huancavelica & 494963 & 81 & 16,4 & 75 & 15,2 & 50 & 10,1 & 5 & 1,0 \\
\hline Cajamarca & 1529755 & 217 & 14,2 & 202 & 13,2 & 113 & 7,4 & 10 & 0,7 \\
\hline PERÚ & 31151643 & 30988 & 99,5 & 27299 & 87,6 & 16927 & 54,3 & 1162 & 3,7 \\
\hline
\end{tabular}

Fuente: ESNPCT - MINSA, orden descendente de acuerdo a tasas de incidencia

La tasa de morbilidad se refiere al total de casos de TB (nuevos y antes tratados) notificados en el 2015 por la ESNPCT por cada 100 mil habitantes. La tasa de incidencia se refiere al total de casos nuevos de TB notificados en el 2015 por la ESNPCT por cada 100 mil habitantes

La tasa de TB pulmonar frotis positivos se refiere al total de casos de TB pulmonar frotis positivos notificados en el 2015 por la ESNPCT por cada 100 mil habitantes

La tasa de mortalidad corresponde al total de muertes durante el tratamiento anti-TB notificados por la ESNPCT en el 2015 por cada 100 mil habitantes 
Tabla 2. Distribución proporcional de casos nuevos de TB según edad y género, Perú 2015

\begin{tabular}{lcccc}
\hline $\begin{array}{l}\text { Grupo de } \\
\text { edad }\end{array}$ & $\begin{array}{c}\text { Casos } \\
\text { nuevos }\end{array}$ & \% total & \% varones & $\%$ mujeres \\
\hline 0 a 4 años & 273 & 1 & 50 & 50 \\
\hline 5 a 14 años & 1092 & 4 & 51 & 49 \\
15 a 24 años & 7917 & 29 & 63 & 37 \\
25 a 34 años & 6006 & 22 & 61 & 39 \\
35 a 44 años & 4095 & 15 & 60 & 40 \\
45 a 54 años & 2730 & 10 & 62 & 38 \\
55 a 64 años & 2183 & 8 & 61 & 39 \\
65 a más años & 3003 & 11 & 60 & 40 \\
\hline Total & 27299 & 100 & 61 & 39 \\
\hline
\end{tabular}

proporción de casos (29\%) y la población económicamente activa, entre 15 y 64 años, constituye el $84 \%$.

\section{LA TB RESISTENTE A MEDICAMENTOS}

De acuerdo a la Norma Técnica de Tuberculosis del Perú, la TB resistente a medicamentos (TB DR) se clasifica de la siguiente manera: i) TB resistente a isoniacida $(\mathrm{H})$, si la resistencia es a isoniacida, pero no a rifampicina; ii) TB resistente a rifampicina $(R)$, si la resistencia es a rifampicina, pero no isoniacida; iii) TB multidrogorresistente (TB MDR), si la resistencia es a $H$ y $R$, y iv) TB extensamente resistente (TB XDR), si además de $H$ y $R$, es resistente a una fluoroquinolona y a un inyectable de segunda línea ${ }^{(11)}$.
En la Figura 2 se muestra la tendencia de las cuatro formas de TB resistente notificada por la ESNPCT entre los años 2008 y 2015. Asimismo, se presenta el incremento de las pruebas de sensibilidad rápida (PSR) para detectar resistencia a H y R por los métodos Genotype ${ }^{\circledR}$ MTBDRplus ${ }^{(12)}$ nitrato reductasa (Griess) y MODS (Microscopic Observation Drug Susceptibility), este último desarrollado en el Perú por Caviedes et al. ${ }^{(13)}$. El incremento significativo de las PSR en el Perú, de 2636 pruebas en el 2008 a 26500 en el 2015, ha contribuido con la detección precoz de un mayor número de casos de TB DR y en el incremento de pacientes que han tenido acceso oportuno a medicamentos de segunda línea. En la Tabla 3 se presenta el número de casos de TB DR por región de salud para el año 2015. Lima Metropolitana y el Callao notificaron el $70,2 \%$ de TB MDR y el $75 \%$ de los casos de TB XDR.

\section{COINFECCIÓN TB-VIH}

La infección por el VIH en el Perú en población general es menor al $1 \%$ y la epidemia es "concentrada" en el grupo de hombres que tienen sexo con otros hombres y transgénero femeninas (prevalencia de VIH en el estudio del año 2011 fue 12,4\%) ${ }^{(14)}$. La cobertura del tamizaje de VIH en personas con TB se ha incrementado de $18,4 \%$ en el 2012 a $79,6 \%$ en el 2015 debido a un mayor acceso a las pruebas rápidas de diagnóstico para VIH. A nivel nacional, la proporción de personas con TB/VIH fue de $4,9 \%$ en el año 2015 . Proporciones superiores

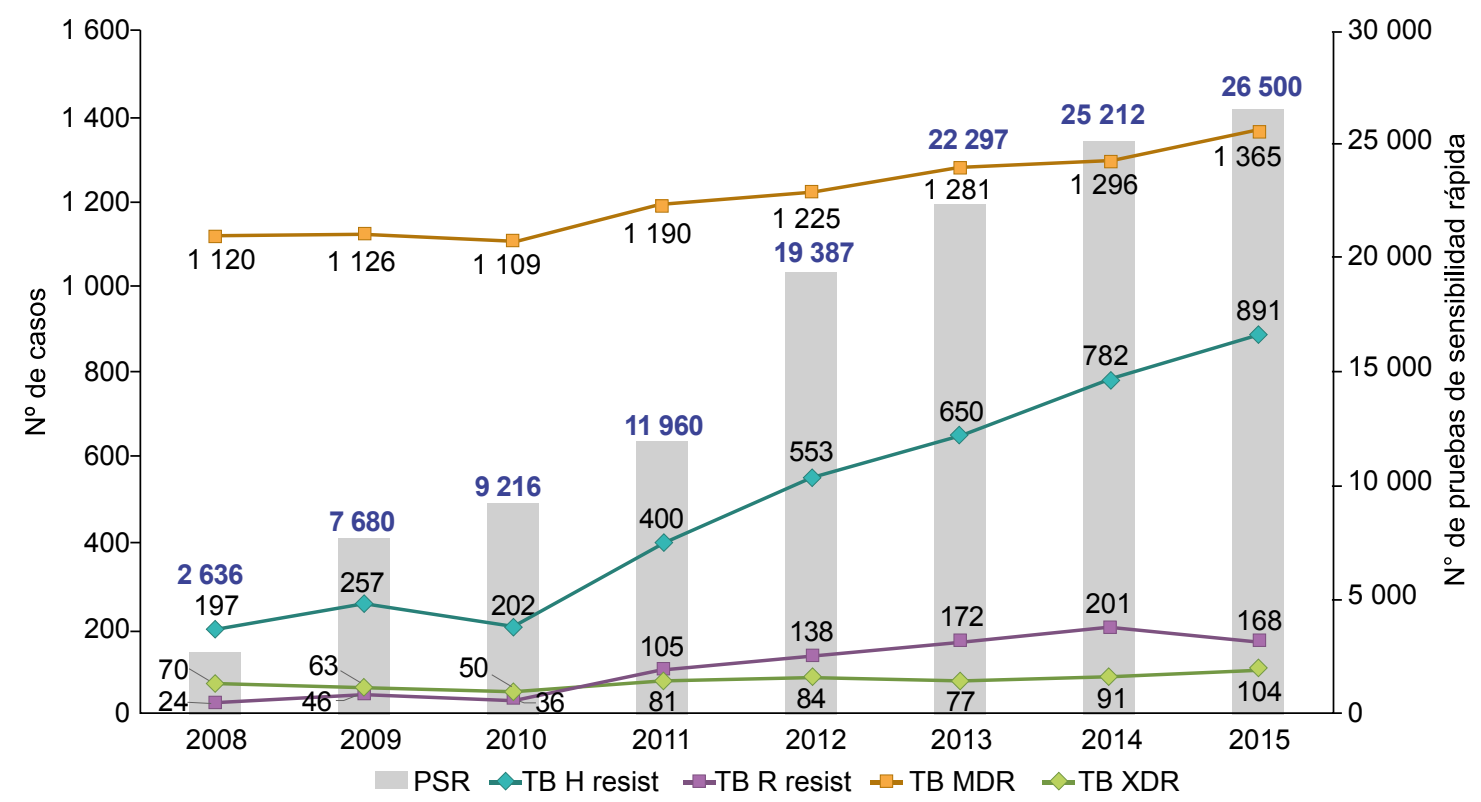

PSR: Pruebas de sensibilidad rápida; TB H resist: Tuberculosis resistente a isoniacida; TB R resist: Tuberculosis resistente a rifampicina; TB MDR: Tuberculosis multidrogorresistente; TB XDR: Tuberculosis extensamente resistente

Figura 2. Casos notificados de TB resistente a medicamentos y pruebas de sensibilidad rápida (PSR), Perú 2008 2015 Fuente: ESNPCT - MINSA 
Tabla 3. Tuberculosis resistente a medicamentos según región de salud Perú, 2015.

\begin{tabular}{|c|c|c|c|c|c|c|c|c|}
\hline \multirow{2}{*}{ Región de Salud } & \multicolumn{2}{|c|}{ TB H Resistente } & \multicolumn{2}{|c|}{ TB R Resistente } & \multicolumn{2}{|c|}{ TB MDR } & \multicolumn{2}{|c|}{ TB XDR } \\
\hline & $\mathbf{N}$ & $\%$ & $\mathbf{N}$ & $\%$ & $\mathbf{N}$ & $\%$ & $\mathbf{N}$ & $\%$ \\
\hline Lima Metropolitana & 506 & 56,8 & 104 & 61,9 & 858 & 62,8 & 65 & 62,5 \\
\hline Lima Provincia & 73 & 8,2 & 13 & 7,7 & 170 & 12,4 & 10 & 9,6 \\
\hline Callao & 86 & 9,7 & 14 & 8,3 & 101 & 7,4 & 13 & 12,5 \\
\hline La Libertad & 80 & 9,0 & 4 & 2,4 & 43 & 3,1 & 2 & 1,9 \\
\hline Ucayali & 8 & 0,9 & 4 & 2,4 & 33 & 2,4 & 1 & 1,0 \\
\hline Ancash & 22 & 2,5 & 3 & 1,8 & 30 & 2,2 & 3 & 2,9 \\
\hline Ica & 11 & 1,2 & 3 & 1,8 & 26 & 1,9 & 4 & 3,8 \\
\hline Junín & 12 & 1,3 & 4 & 2,4 & 18 & 1,3 & 1 & 1,0 \\
\hline Madre de Dios & 9 & 1,0 & 1 & 0,6 & 14 & 1,0 & 0 & 0,0 \\
\hline Huánuco & 12 & 1,3 & 6 & 3,6 & 13 & 1,0 & 0 & 0,0 \\
\hline Piura & 17 & 1,9 & 0 & 0,0 & 12 & 0,9 & 1 & 1,0 \\
\hline Arequipa & 12 & 1,3 & 2 & 1,2 & 9 & 0,7 & 1 & 1,0 \\
\hline Lambayeque & 8 & 0,9 & 2 & 1,2 & 8 & 0,6 & 1 & 1,0 \\
\hline Cusco & 6 & 0,7 & 2 & 1,2 & 7 & 0,5 & 0 & 0,0 \\
\hline Moquegua & 0 & 0,0 & 0 & 0,0 & 5 & 0,4 & 0 & 0,0 \\
\hline Loreto & 0 & 0,0 & 1 & 0,6 & 4 & 0,3 & 0 & 0,0 \\
\hline San Martín & 1 & 0,1 & 0 & 0,0 & 4 & 0,3 & 0 & 0,0 \\
\hline Tacna & 16 & 1,8 & 1 & 0,6 & 4 & 0,3 & 0 & 0,0 \\
\hline Cajamarca & 1 & 0,1 & 1 & 0,6 & 3 & 0,2 & 0 & 0,0 \\
\hline Puno & 1 & 0,1 & 0 & 0,0 & 2 & 0,1 & 0 & 0,0 \\
\hline Apurímac & 1 & 0,1 & 0 & 0,0 & 1 & 0,1 & 1 & 1,0 \\
\hline Ayacucho & 7 & 0,8 & 2 & 1,2 & 1 & 0,1 & 1 & 1,0 \\
\hline Amazonas & 0 & 0,0 & 0 & 0,0 & 0 & 0,0 & 0 & 0,0 \\
\hline Huancavelica & 1 & 0,1 & 0 & 0,0 & 0 & 0,0 & 0 & 0,0 \\
\hline Pasco & 1 & 0,1 & 1 & 0,6 & 0 & 0,0 & 0 & 0,0 \\
\hline Tumbes & 0 & 0,0 & 0 & 0,0 & 0 & 0,0 & 0 & 0,0 \\
\hline PERÚ & 891 & 100,0 & 168 & 100,0 & 1366 & 100,0 & 104 & 100,0 \\
\hline
\end{tabular}

Fuente: ESNPCT - MINSA, orden descendente de acuerdo a proporción de TB MDR

TB H resistente: Tuberculosis resistente a isoniacida; TB R resistente: Tuberculosis resistente a rifampicina; TB MDR: Tuberculosis multidrogorresistente; TB XDR: Tuberculosis extensamente resistente; Lima Metropolitana: Lima Ciudad + Lima Este + Lima Sur.

fueron alcanzadas por la región Loreto con 9,4\%, Callao $7,4 \%$, Tumbes $6,1 \%$, San Martin y Lambayeque 5,9\%, Ancash y Lima Metropolitana 5,1\% (Figura 3).

\section{TUBERCULOSIS Y DIABETES MELLITUS}

La prevalencia de diabetes mellitus (DM) en el Perú, se estima en $7,0 \%$ (IC $95 \% 5,3 \%$ a $8,7 \%$ ) y $8,4 \%$ (IC $95 \%$ $5,6 \%$ a $11,3 \%$ ) en Lima Metropolitana ${ }^{(15)}$. La norma nacional dispone que toda persona con TB debe ser evaluada para descartar DM mediante la determinación de la glicemia en ayunas. El costo de este examen y otros exámenes auxiliares, basales y de seguimiento, son cubiertos por diferentes seguros de salud en el país. La cobertura de tamizaje de DM en pacientes con TB se ha incrementado de $37,8 \%$ en el 2012 a $68 \%$ en el 2015 . La prevalencia de DM en personas con TB a nivel nacional fue de $5,9 \%$. Las regiones con mayor prevalencia de comorbilidad TB DM fueron Loreto 12,1\%; Madre de Dios y Ucayali 8,8\%; Ancash 8,7\%; La Libertad 8,6\%; Tumbes $8,4 \%$; Piura $8,3 \%$; Lambayeque $6,2 \%$, y Callao $5,8 \%$.

\section{TUBERCULOSIS EN PERSONAS PRIVADAS DE LIBERTAD}

Según el INPE, para el año 2015 las personas privadas de libertad (PPL) sumaron 75003 en 67 establecimientos penitenciarios a nivel nacional. Entre los años 2013 y 2015, el INPE incrementó la notificación de casos de TB, de 780 casos en el 2011, a 2155 en el 2015, que corresponde a una tasa de 2643 casos por cada 100 mil PPL. Este incremento se asoció a una mayor identificación de sintomático respiratorios, de 23011 en el año 2011 a 35659 en el 2015. Con relación al diagnóstico de TB DR, a través de la prueba Genotype MTBDRplus, en el 2015, se notificaron 205 casos confirmados de TB MDR y cinco casos de TB XDR. Los establecimientos penitenciarios que concentran el mayor número de casos de TB están 


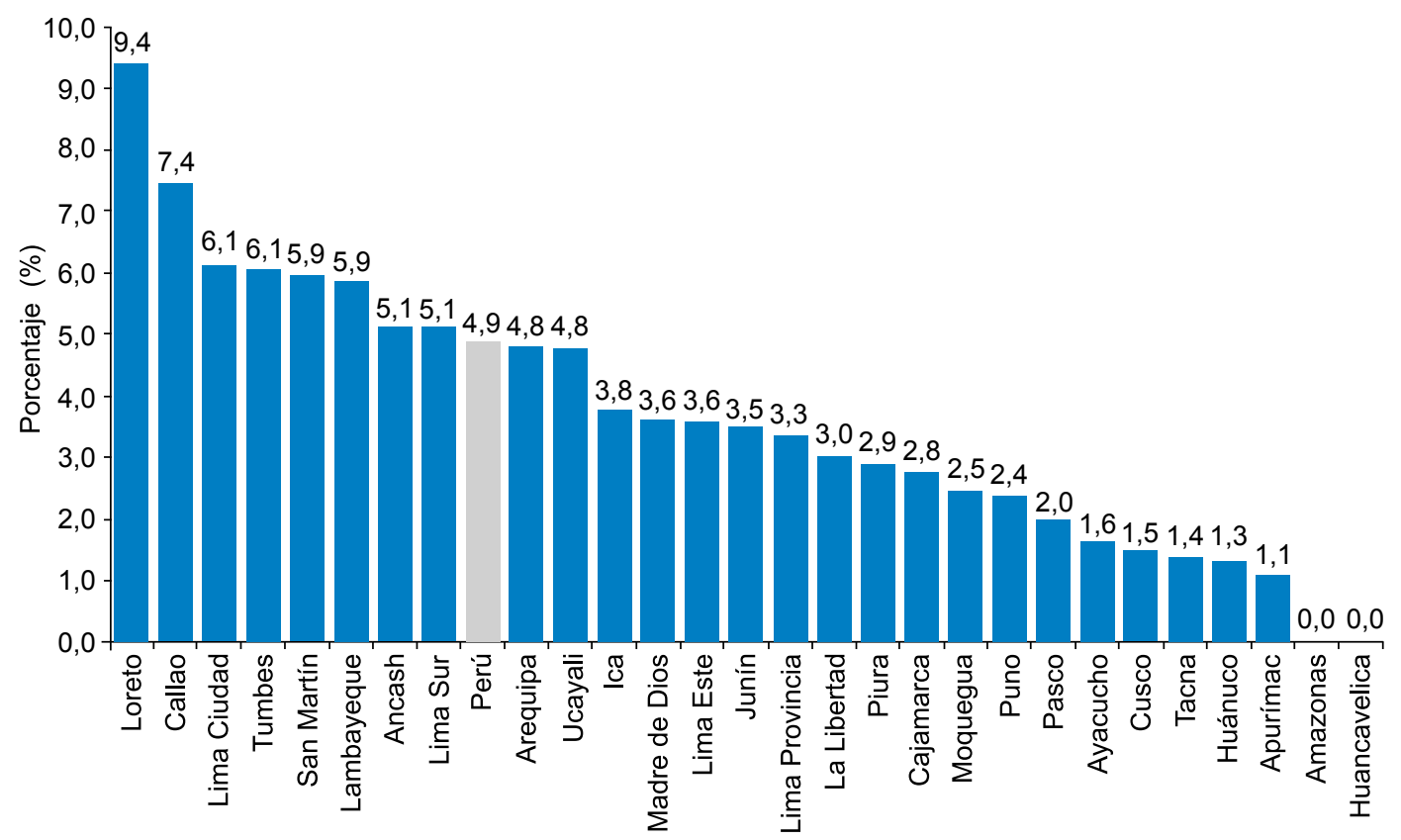

Figura 3. Prevalencia de la coinfección TB-VIH según regiones de salud de Perú, 2015. Fuente: ESNPCT - MINSA

ubicados en la jurisdicción de la Oficina Regional Lima (Lima Metropolitana, Callao y Lima Provincias) con el $79 \%$ de la carga de TB, seguido de la Oficina Regional Norte Chiclayo con el $13 \%$.

\section{TUBERCULOSIS EN LA SEGURIDAD SOCIAL}

El sistema de seguridad social en el Perú, EsSalud, atiende a 10,8 millones de afiliados a nivel nacional (16) En el año 2015, EsSalud notificó 5559 casos de TB, que corresponde al $18,5 \%$ del total de casos en el año 2015. El 3,9\% de los casos de TB en EsSalud tuvo co- infección con el VIH y el 9\% tuvo co-morbilidad con DM. En los últimos 5 años, la tasa de incidencia en EsSalud se mantiene alrededor de 48 casos por 100 mil asegurados.

\section{TUBERCULOSIS INFANTIL}

Se define como TB infantil cuando afecta a menores de 15 años. En la Figura 4 se presenta la proporción de casos nuevos de TB pulmonar infantil con respecto al total de casos nuevos que fueron notificados cada año al MINSA. Se aprecia que la proporción de TB infantil ha disminuido de 7,9 a $5,2 \%$ entre los años 2008 y 2015 . Con respecto

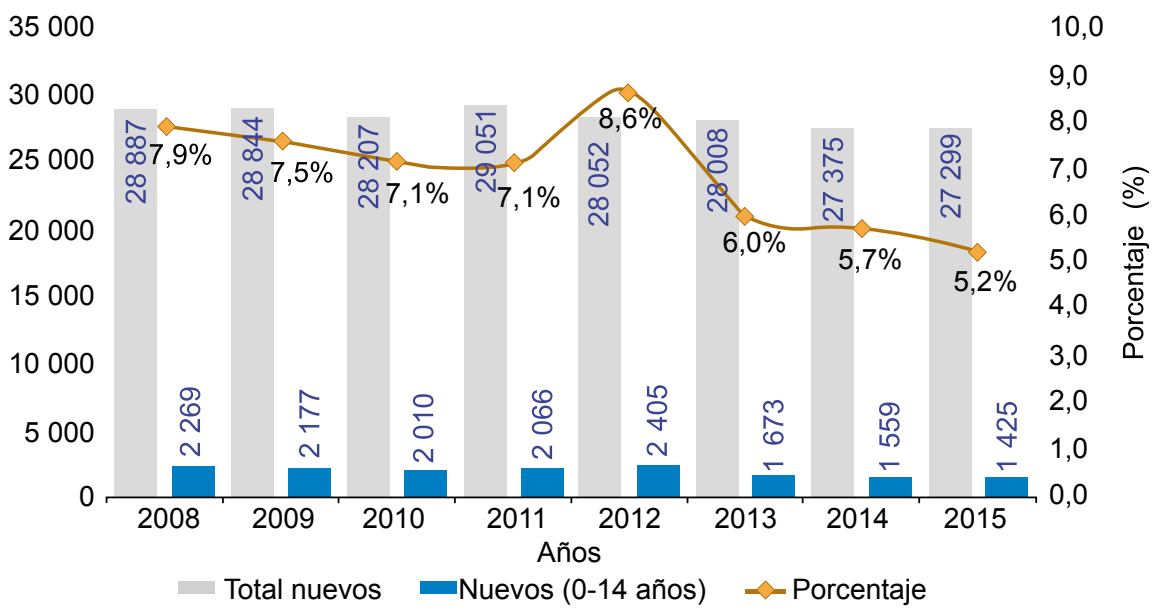

Fuente: ESNPCT-2015

Figura 4. Proporción de TB infantil en casos nuevos de TB, Perú 2008 - 2015 
a los casos de TB MDR confirmados con pruebas de sensibilidad, se evidencia también una disminución en la proporción de casos de TB MDR infantil con respecto al total de casos de TB MDR, de 3,1\% (34 casos) en el año 2010 a 1\% (14 casos) en el año 2015 (Figura 5). En las figuras 4 y 5 se puede observar una disminución sostenida en el número de casos de TB infantil lo que hace menos probable que se deba a un error en la información consignada por la ESNPCT. Una mayor detección de casos de TB activa, el diagnóstico precoz de la TB MDR mediante PSR y el inicio oportuno de tratamiento adecuado, contribuye con la disminución de la transmisión de la TB de personas adultas a los niños. Sin embargo, se requieren estudios específicos que confirmen esta reducción y evalúen sus causas.

\section{TUBERCULOSIS EN TRABAJADORES DE SALUD}

La TB es una enfermedad ocupacional y de notificación obligatoria en el Perú (17). En los últimos 5 años ha disminuido la incidencia de TB en el personal de salud, de 215 casos en el año 2011 a 126 casos en el año 2015. Esta disminución también ha sido mayor en los casos de TB resistente a medicamentos, de 46 casos en el 2007 a 6 casos en el 2015. Esta reducción de casos puede deberse al mejor reporte operado en los últimos años, que evita la duplicidad de notificaciones por MINSA y la Seguridad Social, y al efecto de la implementación de planes de control de infecciones por TB en los principales hospitales del país. Del total de casos de TB en personal de salud notificados en el 2015 , el $57 \%$ pertenecieron al MINSA, $36 \%$ a EsSalud y $7 \%$ a otras instituciones.

\section{AVANCES EN EL CONTROL DE LA TB ENTRE LOS AÑOS 2011 Y 2015}

Entre los años 2011 y 2015, se han logrado avances en el control de la TB que pueden categorizarse en tres enfoques: biomédico, de gestión pública y de determinantes sociales de la salud.

\section{ENFOQUE BIOMÉDICO}

El país ha logrado cumplir los Objetivos de Desarrollo del Milenio (ODM) con respecto a la reducción de la TB. Según el Reporte Global de la Tuberculosis de la OMS para el año $2015^{(2)}$, se ha logrado reducir en $52 \%$ el número de casos nuevos de personas que contraen anualmente esta enfermedad, pasando de 52715 en 1990 a 27412 casos en el 2015. De igual manera, se ha reducido en $74 \%$ el número de fallecidos por TB, pasando de 2931 en 1990 a 1237 en el 2015.

Como avance significativo, se destaca la actualización de la Norma Técnica de Salud (NTS) en el año 2013, para lo cual se realizaron reuniones técnicas para la elaboración, validación y aprobación de la "Norma Técnica de Salud 104 para la Atención Integral de las Personas Afectadas por Tuberculosis", la que ha sido implementada por todos los subsectores de salud del país ${ }^{(11)}$.

Dentro de las innovaciones dispuestas en la NTS 104, en el marco del enfoque biomédico, se pueden citar las siguientes:

- Notificación obligatoria de la TB en el territorio nacional en todos los sistemas de salud, publico, privados y mixtos.

- Estratificación de las regiones geográficas en escenarios epidemiológicos, de acuerdo al riesgo de transmisión de la TB, con el objetivo de priorizar los recursos económicos a asignar.

- Aplicación de recomendaciones internacionales para el control de infecciones por TB y bioseguridad.

- Priorización de las indicaciones de la terapia preventiva con isoniacida (TPI), en grupos de mayor riesgo.

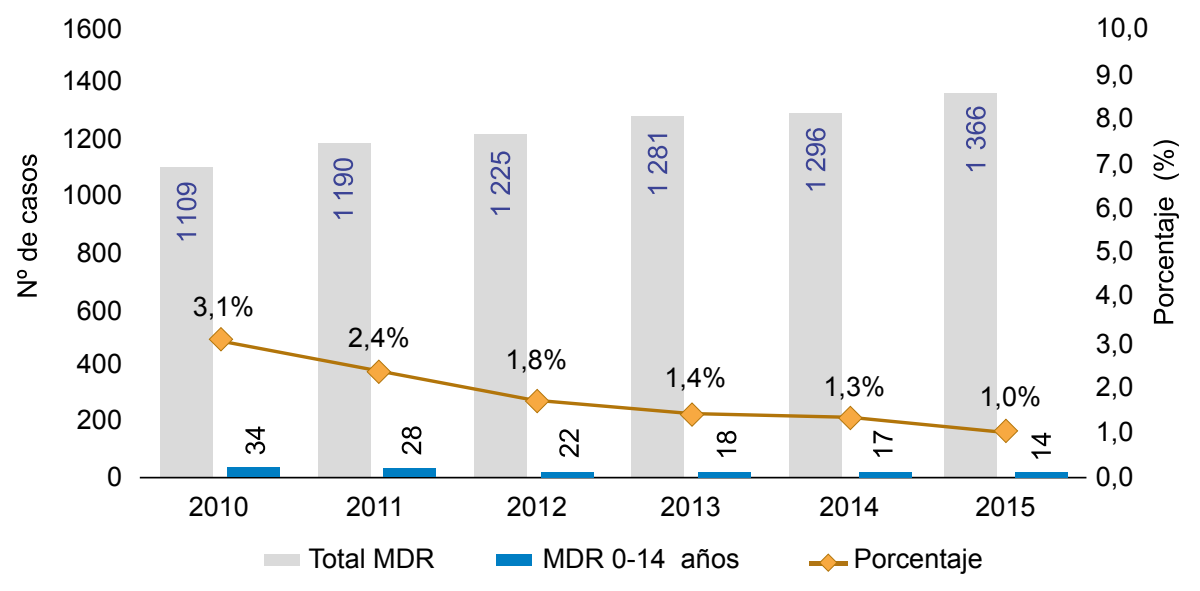

Figura 5. Proporción de casos de TB MDR confirmados con prueba de sensibilidad en población infantil, Perú 2010 - 2015 Fuente: ESNPCT- MINSA 
- Tamizaje de TB con dos muestras de esputo en el mismo día, en personas con alto riesgo de no retornar al establecimiento de salud, lo cual no afecta la sensibilidad de la baciloscopía, especialmente en salas de emergencia o para población en tránsito ${ }^{(18)}$. - Incorporación de la identificación del complejo M. tuberculosis y de micobacterias no tuberculosas con métodos de biología molecular en aislamientos de personas con $\mathrm{VIH}$ y en cepas resistentes a medicamentos.

- Implementación de exámenes auxiliares basales financiados por seguros de salud, para descartar gestación, coinfección y comorbilidades que permitan una mejor aproximación terapéutica.

- Implementación del acceso universal a pruebas rápidas directas (a partir de esputo), para detectar resistencia a isoniacida y rifampicina por las pruebas MODS y prueba de sondas de ADN en línea (Genotype MTBDRplus).

- Adjudicación al sistema NETLAB del INS, como único medio para acceder al resultado de las pruebas de laboratorio de tuberculosis. El NETLAB (www. netlab.ins.gob.pe) es un sistema en plataforma Web, accesible desde cualquier punto de atención a nivel nacional, mediante claves de seguridad.

- Estandarización de los esquemas de tratamiento de la TB de acuerdo a los cuatro posibles perfiles de resistencia a isoniacida y rifampicina: i) sensible a isoniacida y rifampicina; ii) resistente a isoniacida; iii) resistente a rifampicina, y iv) TB-MDR. Esta clasificación se basa en el perfil de resistencia y la sensibilidad de más de 10 mil cepas de $M$. tuberculosis evaluadas en el INS de Perú ${ }^{(19)}$. En la Figura 6 se presenta el algoritmo de los nuevos esquemas de tratamiento anti-TB en el Perú, de acuerdo al correcto uso de las pruebas de sensibilidad rápida.

- Nueva clasificación de los esquemas de tratamiento en dos grupos: i) esquemas para TB sensible, y ii) esquemas para TB resistente a medicamentos, eliminando la anterior nomenclatura de esquema I, esquema II, esquema IV.

- Fortalecimiento del tratamiento de la TB sensible y la TB resistente. En la Tabla 4 se resumen las principales innovaciones dispuestas para fortalecer los esquemas de tratamiento para la TB sensible y la TB resistente, y en la Tabla 5 se presentan las modificaciones de la duración y posología de los esquemas para TB sensible según edad, condición $\mathrm{VIH}$ y compromiso extrapulmonar.

- Estandarización y optimización de los tiempos de diagnóstico e inicio de tratamiento para la TB sensible y la TB resistente y simplificación del proceso de atención para acceder a medicamentos de segunda línea, a través de los Comités de Retratamiento Regionales y Nacional.

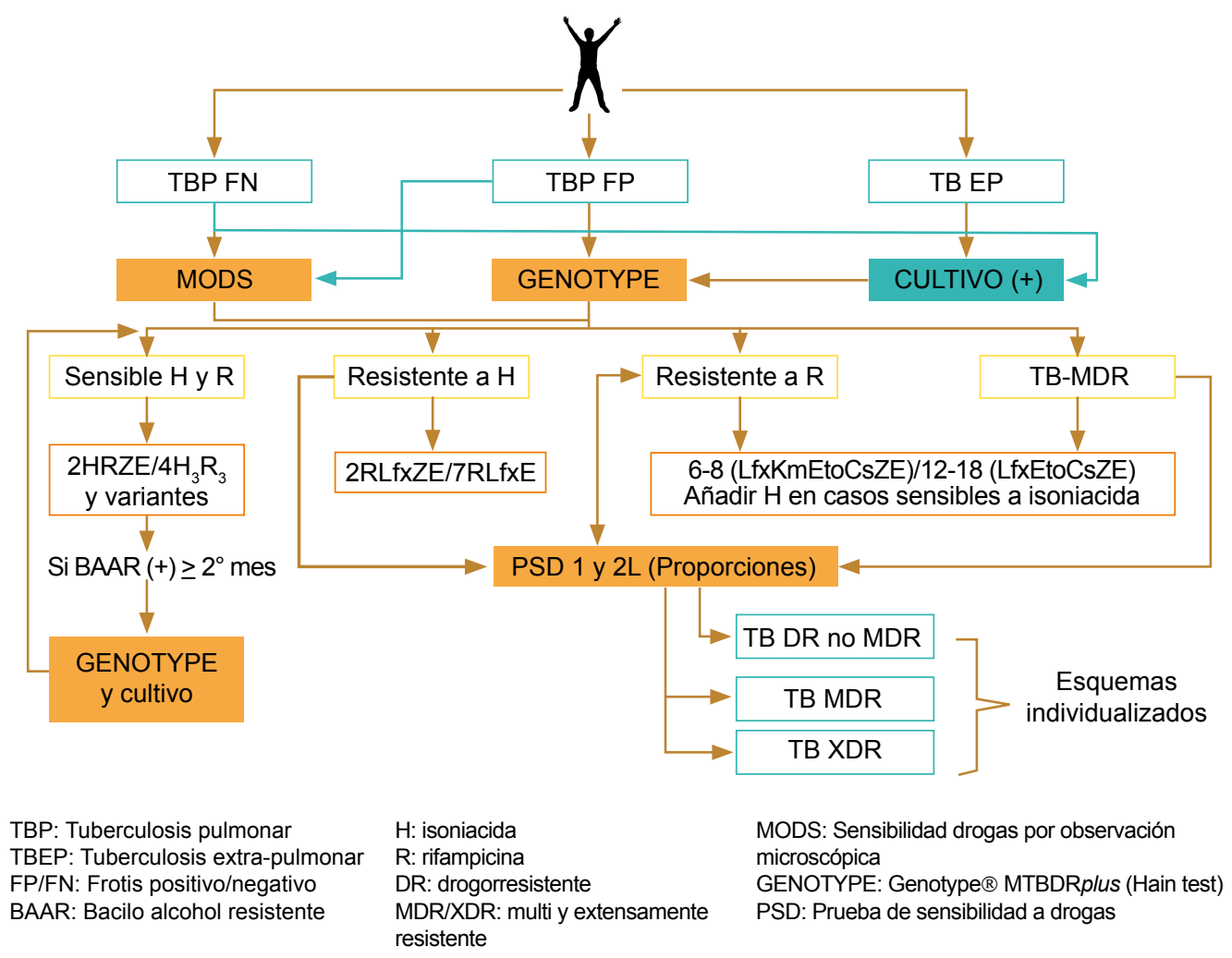

Figura 6. Algoritmo de tratamiento de la TB en el Perú según susceptibilidad a isoniacida y rifampicina. fuente: adaptado de Norma Técnica de Salud 104- Minsa 
Tabla 4. Innovaciones para el fortalecimiento del tratamiento de la TB sensible y TB resistente a medicamentos, según Norma Técnica de Salud 104

\begin{tabular}{|c|c|}
\hline Tratamiento TB sensible & Tratamiento TB resistente \\
\hline $\begin{array}{l}\text { - Incorporación de la tercera dosis en la segunda fase (fase } \\
\text { de esterilización) del esquema para TB sensible, para reducir } \\
\text { las recaídas. } \\
\text { - Incorporación de esquemas de tratamiento diarios para } \\
\text { pacientes con VIH o compromiso del sistema nervioso central } \\
\text { o del sistema osteoarticular. } \\
\text { Incorporación en el petitorio nacional de la presentación } \\
\text { farmacéutica de los medicamentos de primera línea en dosis } \\
\text { fijas combinadas (DFC). } \\
\text { - Evaluación con la prueba Genotype MTBDRplus a todos } \\
\text { los casos con baciloscopía positiva a partir del segundo mes } \\
\text { de tratamiento, para la detección de resistencia. } \\
\text { Implementación del cultivo al inicio y término del tratamiento } \\
\text { para confirmar la etiología y la curación bacteriológica, } \\
\text { respectivamente. } \\
\text { - Estandarización de inicio de tratamiento dentro de las } 24 \\
\text { horas posteriores al diagnóstico. }\end{array}$ & $\begin{array}{l}\text { - Implementación de esquemas de tratamiento basados en los } \\
\text { perfiles de resistencia inicial a isoniacida y rifampicina. } \\
\text { Implementación del esquema 2RLfxEZ/7RLfXE para la TB } \\
\text { resistente a INH no MDR } \\
\text { - Elaboración de esquemas individualizados a partir de la prueba } \\
\text { de sensibilidad convencional a medicamentos de primera y } \\
\text { segunda línea. } \\
\text { - Implementación del esquema para la TB extensamente } \\
\text { resistente (TB-XDR) con medicamentos del anterior quinto grupo } \\
\text { de la OMS: linezolid, carbapenems (imipenem o meropenem) } \\
\text { con ácido clavulánico y clofazimina, además de la tioridazina. } \\
\text { - Implementación del manejo programático de la TB XDR } \\
\text { "centrado en el paciente" y no en la disponibilidad de los servicios } \\
\text { de salud: hospitalización, tratamiento en domicilio, etc. } \\
\text { - Estandarización de inicio de tratamiento hasta } 15 \text { días posterior } \\
\text { al diagnóstico de cualquier forma de TB resistente. }\end{array}$ \\
\hline
\end{tabular}

\section{ENFOQUEDEGESTIÓNPÚBLICAYDETERMINANTES SOCIALES}

Con respecto al enfoque de gestión pública, el principal logro en los últimos años ha sido la publicación de la
Ley 30287, "Ley para la Prevención y Control de la Tuberculosis en el Perú", ${ }^{(6)}$ y su reglamento ${ }^{(7)}$, en un trabajo coordinado entre el Congreso de la República, el Ministerio de Salud y la sociedad civil organizada. Esta Ley, según comunicaciones personales de funcionarios

Tabla 5. Esquemas de tratamiento para tuberculosis sensible en el Perú según Norma Técnica de Salud 104

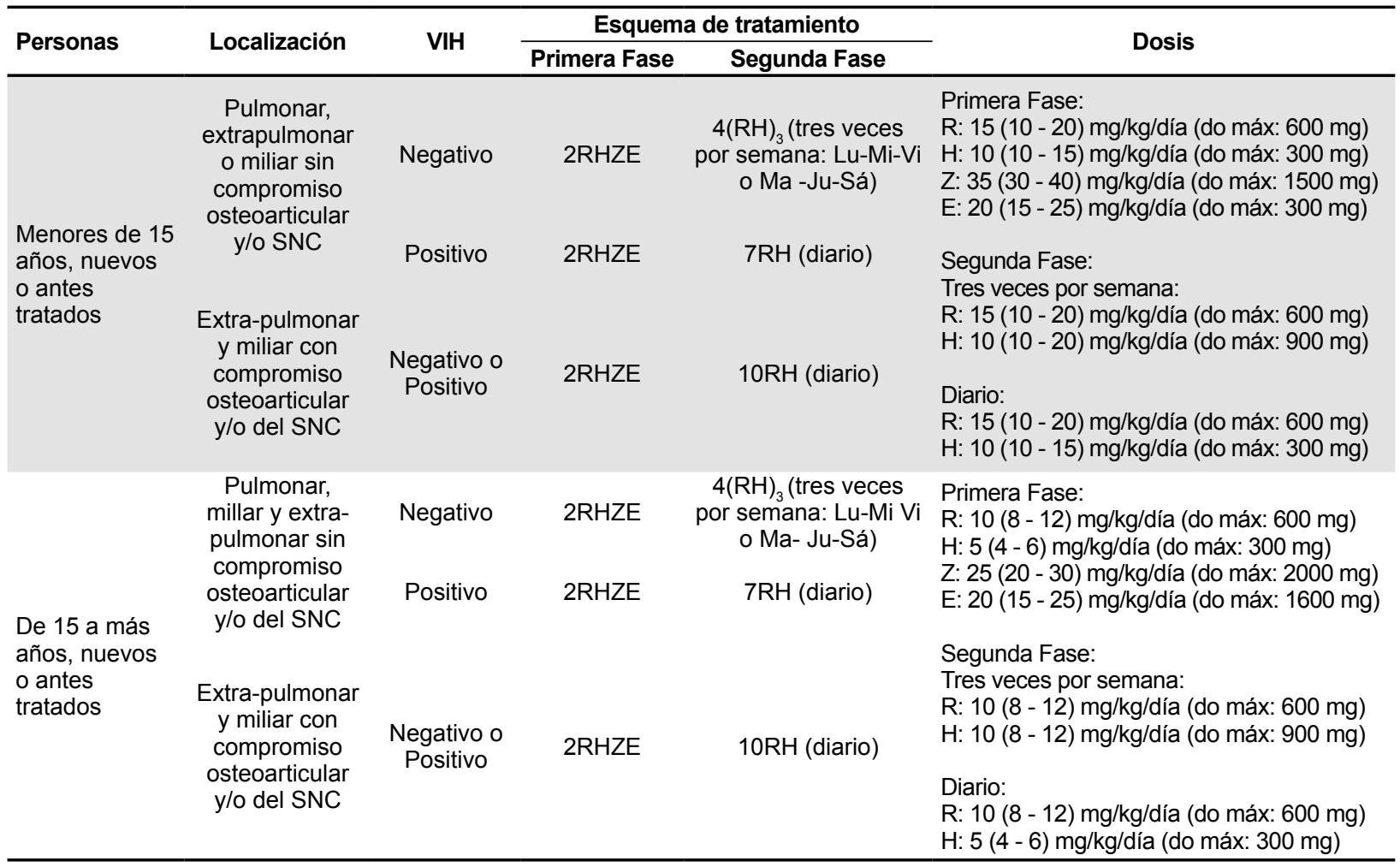


de la OPS, constituye un modelo para la región de las Américas, en cuanto a políticas sociales para abordar el problema de la TB.

La Ley 30287 dispone la elaboración de un plan de emergencia para el control de la TB en las áreas de mayor prevalencia, por lo que, en el año 2015, la ESNPCT elaboró el "Plan de Emergencia para el control de la TB en Lima Metropolitana y Callao", que fue aprobado por el MINSA con RM 193-2015/MINSA, mediante el cual se ha priorizado el fortalecimiento de los servicios de TB de los establecimientos de salud con mayor carga a nivel de Lima y Callao. Asimismo, EsSalud, el INPE y la Región Callao, basados en este plan, han desarrollado sus respectivos planes para el control de la TB.

La citada Ley también dispone el incremento y la intangibilidad del financiamiento asignado a la lucha contra la TB y dispone que los siguientes Ministerios: Salud, Trabajo y Promoción del Empleo; Justicia y Derechos Humanos; Educación; Interior; Defensa; Vivienda, Construcción y Saneamiento; Desarrollo e Inclusión Social; Transportes y Comunicaciones; Mujer y Poblaciones Vulnerables, y Producción; así como la Municipalidad Metropolitana de Lima y los gobiernos regionales y locales a nivel nacional, asuman las actividades y financiamiento directo para abordar las determinantes sociales de salud relacionadas a la TB ${ }^{(6)}$.

Otro avance notable ha sido el reordenamiento del financiamiento de las actividades de control y prevención de la TB en el país. Se ha logrado la consolidación de la programación y ejecución del Programa Presupuestal $016 \mathrm{~TB} / \mathrm{VIH}$, modalidad de presupuesto por resultado $(\mathrm{PpR})$, que está orientado a disminuir la incidencia de la TB y de la infección por el VIH/SIDA. Comprende un conjunto de intervenciones de detección, diagnóstico, tratamiento, actividades de promoción de la salud, enmarcados en 11 productos presupuestales. EI Seguro Integral de Salud (SIS), seguro creado en el año 2002 para proteger la salud de los peruanos que no cuentan con otro seguro de salud, priorizando a familias en situación de pobreza y pobreza extrema, $y$ otras instituciones administradoras de fondos de aseguramiento en salud (IAFAS) complementan el financiamiento para las actividades de prevención y control de la TB.

Recientemente, los ministerios de Defensa y del Interior han logrado incorporar financiamiento del Programa Presupuestal 016 TB/VIH. El reordenamiento presupuestal de la ESNPCT evita la duplicidad de financiamiento con el SIS y asigna la fuente de los fondos para cada actividad de control y prevención, lo que permite una mejor programación y monitoreo del gasto y del impacto de las intervenciones financiadas.
Por otro lado, se ha desarrollado e implementado el Sistema de Información Gerencial de la TB en el Perú (Sistema SIG-TB), sistema Web que permite el registro de todos los casos de TB en el país, reemplazando los registros manuales y posibilitando bases nominales con información de cada caso y no información agregada. Este sistema se interconecta a la base de datos del Registro Nacional de Identificación (RENIEC) y a la base de datos del Registro Nacional de Establecimientos de Salud (RENAES), lo que permite identificar la localización geográfica donde fue notificado el caso. La información generada por el SIG-TB, permite tener información en tiempo real para la toma de decisiones en los diferentes niveles de gestión del Ministerio de Salud y otras instituciones (INPE, EsSalud, FF.AA. y Policía Nacional).

Se ha logrado conformar la Red de Investigación en TB, que incorpora instituciones públicas, académicas, organismos no gubernamentales y sociedades científicas, que trabajan en TB. La finalidad de la Red es promover la investigación y direccionar los estudios en base a la necesidad de la ESNPCT. Como producto de esta Red, se han desarrollado cuatro jornadas científicas: "Contribución Peruana para el Control de la Tuberculosis" donde se presentaron los resultados obtenidos de los estudios hechos en el país y las nuevas evidencias para actualizar los conocimientos del personal de salud que laboran en TB, en los diferentes niveles de atención.

Entre otros avances logrados en gestión y abordaje de determinantes en los últimos cinco años, podemos mencionar: i) lograr la afiliación al Seguro Integral de Salud del $89 \%$ de la población penitenciara en el Perú, gracias a un trabajo coordinado con el Ministerio de Justicia- INPE, incluyendo el desarrollo de 30 campañas de salud en las que se realizó atención especializada e integrada de TB y $\mathrm{VIH}$ en 24 establecimientos penitenciarios; ii) coordinación y desarrollo de guías metodológicas de capacitación para el control de TB en el trabajo, elaboración de material educativo e informativo para prevenir la TB en los centros laborales, liderado por el Ministerio de Trabajo; (20) iii) coordinación con el Ministerio de Educación para incorporación, en el contenido curricular de Educación Básica Regular, de los tópicos sobre prevención de la TB en instituciones educativas públicas y privadas, y iv) aprobación de la Directiva Sanitaria Administrativa que actualiza el contenido de la canasta de alimentos mensuales para los afectados por TB, incluida en el Programa de Complementación Alimentaria (PCA) del Ministerio de Desarrollo e Inclusión Social (MIDIS), que incrementa el número de raciones por familia, de 2 a 4 contactos, más el caso índice. El financiamiento es asignado por el Ministerio de Economía y Finanzas (MEF) del Perú asciende a más 70 millones de soles y la responsabilidad 
del desarrollo del programa está a cargo del MIDIS en coordinación con el MINSA y los municipios, quienes son los ejecutores finales ${ }^{(21)}$.

\section{DESAFÍOS PARA HACER FRENTE A LA TB EN PERU}

La Asamblea Mundial de Salud aprobó la "Estrategia Fin de la Tuberculosis" en mayo de 2014. El Perú, como miembro de la ONU, también ha suscrito el cumplimiento de esta Estrategia. En el marco de los tres pilares de la "Estrategia Fin de la Tuberculosis" y la Ley 30287, planteamos los siguientes desafíos, adaptados al contexto nacional, para la consideración de los sucesivos equipos de gestión de la ESNPCT.

\section{PILAR 1. DESAFÍOS EN LA PREVENCIÓN Y ATENCIÓN INTEGRAL DE LA TB CENTRADA EN EL PACIENTE}

- Informar y orientar sobre las medidas de prevención y control de la TB a la población general, con énfasis en las regiones que tienen escenarios epidemiológicos de muy alto y alto riesgo de transmisión de la TB.

- Fortalecer la evaluación longitudinal, y no solo de manera transversal, de los contactos domiciliarios y extradomiciliarios de los casos de TB.

- Implementar, monitorear y evaluar nuevas estrategias de búsqueda activa de casos probables de TB en la comunidad.

- Culminar la expansión de la cobertura de diagnóstico de TB por pruebas de biología molecular para drogas de primera línea e implementar para drogas de segunda línea, para disminuir el tiempo de diagnóstico de TB MDR y TB XDR, y de esta forma prescribir oportunamente el tratamiento más eficiente.

- Consolidar la descentralización del cultivo a las regiones de salud y hospitales nacionales con mayor carga de TB.

- Implementar los nuevos regímenes acortados de 9 a 12 meses para la TB MDR respaldos por la OMS, incorporando nuevos medicamentos $(22,23)$.

\section{PILAR 2. POLÍTICAS AUDACES Y SISTEMAS DE SOPORTE}

- Continuar el aseguramiento universal a fin de evitar los gastos catastróficos para las familias de afectados por TB.

- Propiciar el cumplimiento de la Ley 30287 que garantiza la intangibilidad de los fondos asignados al control de la TB en el Perú.

- Garantizar la incorporación de la persona afectada de TB en los Programas de Desarrollo, Protección Social, Vivienda y Trabajo del Estado.

- Promover la participación activa de las empresas públicas y privadas, mediante alianzas públicoprivadas en las actividades de prevención de la TB en el Perú.

\section{PILAR 3. INVESTIGACIÓN E INNOVACIÓN INTENSIFICADA}

- Implementar políticas de desarrollo de recursos humanos en salud con formación en salud pública e investigación y no solo formación clínico-asistencial en las universidades e institutos de educación superior.

- Continuar la ejecución de las Jornadas Científicas Conmemorativas del Día Mundial de Lucha contra la Tuberculosis, que permita intercambiar experiencias y socializar los resultados de las investigaciones con el personal de salud y tomadores de decisiones.

- Publicar e implementar las líneas de investigación en TB para el periodo 2015-2019, elaborado por el Ministerio de Salud, en el marco de las reuniones convocadas por el Instituto Nacional de Salud durante los años 2014 y 2015 en coordinación con la ESN PCT.

- Priorizar el financiamiento de investigaciones en TB por instituciones públicas y privadas, de acuerdo a las líneas de investigación aprobadas.

- Promover la investigación operativa y clínica en TB en todos los niveles de atención para obtener mejores regímenes de tratamiento y medidas preventivas eficientes.

- Desarrollar, validar y estandarizar nuevos métodos de diagnóstico de TB en el lugar de atención del paciente.

- Continuar con la implementación de políticas de salud pública basadas en los resultados de investigaciones científicas, lo que debe lograrse con una actualización periódica, de la Norma Técnica de Salud de la ESNPCT.

- Desarrollar estudios para medir los costos asumidos por las personas afectadas por TB y sus familias después de ser diagnosticados con TB, para monitorear la meta de cero gastos catastróficos por TB.

\section{CONCLUSIONES}

Durante los años 2011 a 2015 se han logrado importantes avances en el control de la TB en el Perú, con una sostenida reducción de la incidencia de TB y con un mayor compromiso del Estado reflejadas en la Ley 30287 y su Reglamento. Sin embargo, aún hay una agenda pendiente y desafiante para conseguir que, en el año 2035, se declare al Perú como libre de TB, tasa de incidencia menor o igual a 10 casos de TB por cada 100 mil habitantes.

\begin{abstract}
Contribución de los autores: VA, EA, y AMT han participado en la concepción de la idea original, planeamiento, redacción y revisión de todo el manuscrito. CF participo en la elaboración de tablas y figuras y revisión de todo el manuscrito. Todos los autores aprobaron la versión final.
\end{abstract}

Fuente de financiamiento: autofinanciado.

Conflicto de interés: los autores declaran no tener conflictos de interés. 


\section{REFERENCIAS BIBLIOGRÁFICAS}

1. Wingfield T, Tovar MA, Huff D, Boccia D, Saunders MJ, Datta S, et al. Beyond pills and tests: addressing the social determinants of tuberculosis. Clin Med (Lond). 2016;16(Suppl 6):s79-s91. doi: 10.7861/clinmedicine.16-6-s79.

2. World Health Organization. Global Tuberculosis Report 2016 [Internet]. Geneva: WHO; 2016. [Citado el 15 de agosto de 2016]. Disponible en: http://apps.who.int/medicinedocs/ documents/s23098en/s23098en.pdf.

3. Aplicación de la estrategia fin de la TB: aspectos esenciales. Ginebra: Organización Mundial de la Salud; 2016. Disponible en: http://www. who.int/tb/publications/2015/end tb_essential_spanish_web.pdf ?ua $=1$.

4. World Health Organization. The End TB Strategy. Global strategy and targets for tuberculosis prevention, care and control after 2015 [Internet]. Geneva: WHO; 2015. [Citado el 1 de agosto de 2016] Disponible en: http:// www.who.int/tb/strategy/End_TB_ Strategy.pdf ?ua $=1$.

5. Uplekar M, Weil D, Lonnroth K, Jaramillo E, Lienhardt C, Dias HM, et al. WHO's new End TB Strategy. The Lancet.385(9979):1799-801. doi: 10.1016/S0140-6736(15)60570-0.

6. Congreso de la República. Ley $\mathrm{N}^{\circ}$ 30287 Ley de Prevención y Control de la Tuberculosis en el Perú 2015. [Citado el 5 de agosto de 2016]. Disponible en: http://190.223.45.115/newtb/ Archivos/Ley_30287_Ley_TB.pdf.

7. Reglamento de la Ley No30287. Ley de Prevención y Control de la Tuberculosis en el Perú. [Internet]. El Peruano. 15 de mayo del 2016; 587360-8. Disponible en: ftp://ftp2. minsa.gob.pe/normaslegales/2016/ DS_021.pdf

8. Principales causas de mortalidad por sexo Perú, año 2014 [Internet]. Ministerio de Salud, Oficina General de Tecnologías de la Información; 2015. Disponible en: http://www. minsa.gob.pe/estadisticas/estadisticas/ mortalidad/macros.asp?00.

9. Ministerio de Salud - Dirección general de Epidemiología. Carga de enfermedad en el Perú, estimación de los años de vida saludables perdidos 2012 [Internet]. Lima: USAID;
2012. [Citado el 20 de julio de 2016] Disponible en: http://www. dge.gob.pe/portal/docs/tools/ Cargaenfermedad2012.pdf.

10. Ministerio de Salud. Impacto socioeconómico de la Tuberculosis en el Perú 2010: documento tecnico [Internet]. Lima: Ministerio de Salud; 2012.

[Citado el 3 de julio de 2016]. Disponible en: http://bvs.minsa.gob. pe/local/minsa/1820.pdf.

11. Norma Técnica de Salud $\mathrm{N}^{\mathrm{o}}$ 104-MINSA/DGSP-V.01. Norma Técnica de Salud para el Manejo Integral de las Personas Afectadas por Tuberculosis [Internet]. Lima: Ministerio de Salud; 2013. [Citado el 15 de agosto de 2016]. Disponible en: $\quad \mathrm{ftp}: / / \mathrm{ftp} 2 . \mathrm{minsa}$.gob.pe/ normaslegales/2013/RM715_2013_ MINSA.pdf.

12. Asencios L, Galarza M, Quispe $\mathrm{N}$, Vasquez L, Leo E, Valencia E, et al. Prueba molecular Genotype MTBDRplus, una alternativa para la detección rápida de tuberculosis multidrogorresistente. Rev Peru Med Exp Salud Publica. 2012;29(1):92-8.

13. Caviedes L, Lee TS, Gilman RH, Sheen P, Spellman E, Lee EH, et al. Rapid, efficient detection and drug susceptibility testing of Mycobacterium tuberculosis in sputum by microscopic observation of broth cultures. The Tuberculosis Working Group in Peru. J Clin Microbiol. 2000;38(3):1203-8.

14. Ministerio de Salud. Dirección General de Epidemiología. Análisis de situación de la salud del Perú [Internet]. Lima: Ministerio de Salud; 2013. [Citado el 15 de julio de 2016]. Disponible en: http://www.dge.gob.pe/portal/docs/ intsan/asis2012.pdf.

15. Seclen SN, Rosas ME, Arias AJ, Huayta E, Medina CA. Prevalence of diabetes and impaired fasting glucose in Peru: report from PERUDIAB, a national urban population-based longitudinal study. BMJ Open Diabetes Res Care. 2015;3(1):e000110. doi: 10.1136/ bmjdrc-2015-000110.

16. EsSalud. Análisis Ejecutivo a nivel Nacional 2015. Seguridad Social del Perú [Internet]. Lima: EsSalud; 2015. 65p [Disponible en: http://www. essalud.gob.pe/downloads/analisis ejec_n_nacional_2015.pdf.

17. Mendoza-Ticona A. Tuberculosis como enfermedad ocupacional. Rev Peru Med Exp Salud Publica. 2012;29(2):232-6.

18. World Health Organization. Sameday diagnosis of tuberculosis by microscopy: policy statement [Internet]. Geneva: World Health Organization; 2011.

19. Mendoza-Ticona A, Moore DA, Alarcon V, Samalvides F, Seas C. Propuesta de esquemas de tratamiento antituberculosis basados en la susceptibilidad a isoniacida y rifampicina. Rev Peru Med Exp Salud Publica. 2013;30(2):197-204.

20. Implementación de programas de prevención y asistencia relacionados a la tuberculosis en el ámbito laboral [Internet]. Ministerio de Trabajo y Promoción del Empleo; 2012. Disponible en: http://www2.trabajo. gob.pe/el-ministerio-2/sector-trabajo/ dir-gen-de-d-f-s-s-t/tbc-en-el-trabajo/.

21. Programa de Complementación Alimentaria [Internet]. Lima: Ministerio de Desarrolo e Inclusión Social; 2013 [citado el 10 de agosto de 2017]. Disponible en: http://www.midis.gob.pe/ index.php/es/pca.

22. Aung KJ, Van Deun A, Declercq E, Sarker MR, Das PK, Hossain MA, et al. Successful '9-month Bangladesh regimen' for multidrug-resistant tuberculosis among over 500 consecutive patients. Int J Tuberc Lung Dis. 2014;18(10):1180-7. doi: 10.5588/ ijtld.14.0100.

23. World Health Organization. WHO treatment guidelines for drug-resistant tuberculosis, 2016 update. October 2016 revision [Internet].Geneva: WHO; 2016. [citado el 10 de agosto de 2017]. Disponible en: http://apps. who.int/iris/bitstream/10665/25012 5/1/9789241549639-eng.pdf ?ua=1

Correspondencia: Alberto Mendoza Ticona Dirección: Av. Cayetano Heredia 451 - 301. Lima 11, Perú.

Teléfono: (511) 953982909

Correo electrónico:mendozalberto@outlook.com 